\title{
Neutrinoless double $\beta$ decay with small neutrino masses
}

\author{
F. del Águila ${ }^{a}$, A. Aparici ${ }^{b}$, S. Bhattacharya ${ }^{c}$, A. Santamaria ${ }^{* b}$ and J. Wudka ${ }^{c}$ \\ ${ }^{a}$ CAFPE and Departamento de Física Teórica y del Cosmos, Universidad de Granada, \\ E-18071 Granada, Spain \\ ${ }^{b}$ Departament de Física Tè̀rica and IFIC, Universitat de València-CSIC, \\ Dr. Moliner 50, E-46100 Burjassot (València), Spain \\ ${ }^{c}$ Department of Physics and Astronomy, University of California, Riverside CA 92521-0413, USA \\ E-mail: faguilaeugr.es, \\ Alberto.Aparici@uv.es, subhaditya123@gmail.com, \\ Arcadi.Santamariaduv.es, jose.wudka@ucr.edu
}

If the only contribution to neutrinoless double beta decay $(0 \nu \beta \beta)$ are neutrino masses its discovery may be very difficult, especially if neutrinos present a normal hierarchy spectrum. However, this is not the only possibility; $0 v \beta \beta$ can in general produce electrons of either chirality, in contrast with the neutrino induced $0 v \beta \beta$ which predicts two left-handed electrons. Using an effective Lagrangian approach we classify the lepton number violating (LNV) effective operators with two leptons of either chirality but no quarks, ordered according to the magnitude of their contribution to $0 v \beta \beta$. We point out that, for each of the three chirality assignments, $e_{L} e_{L}, e_{L} e_{R}$ and $e_{R} e_{R}$, there is only one LNV operator of the corresponding type to lowest order, and these have dimensions 5, 7 and 9, respectively. Neutrino masses are always induced by these extra operators but can be delayed to one (dimension 7) or two loops (dimension 9). Under the assumption that $0 v \beta \beta$ is dominated by the operators of dimension 7 or 9 we find that the scale of new physics should be relatively low ( $\lesssim 30 \mathrm{TeV}$ ). We also list the SM additions generating these operators upon integration of the heavy modes, and discuss simple realistic examples of renormalizable theories for each case. The phenomenology of a model giving rise to the dimension 9 operator has been analyzed with some detail: if $0 v \beta \beta$ is going to be seen in the next round of experiments, the doubly charged scalars of the model could be seen at the LHC and lepton flavour violating (LFV) rates could be at the reach of foreseen experiments. Moreover neutrino masses, which arise at two loops, display a very particular structure and are strongly constrained, in fact, $\sin ^{2} \theta_{13} \gtrsim 0.008$, when $\mu \rightarrow e e e$ is required to lie below its present experimental limit.

Proceedings of the Corfu Summer Institute 2012 "School and Workshops on Elementary Particle Physics and Gravity"

September 8-27, 2012

Corfu, Greece

${ }^{*}$ Speaker. 


\section{Introduction}

The remarkable observation of neutrino oscillations (see [1] and [2,3] for recent reviews and [4-8] for fits including latest data on $\theta_{13}$ ) has provided the first evidence for neutrino masses. On the other hand, the invisible decay width of the Z-boson tells us that there are only three species of light active neutrinos (lighter than about $45 \mathrm{GeV}$ and interacting with full gauge strength). If lepton number (LN) is conserved, neutrinos are Dirac fields composed by the three active (left-handed) and three sterile (right-handed) neutrinos. In that case, neutrinos are accommodated in the Standard Model (SM) as the charged fermions, and their masses and mixings, like in the quark sector, can be parametrized by the three masses, $m_{1,2,3}$, three mixing angles, $\theta_{12}, \theta_{23}, \theta_{13}$, and one CP-violating phase, $\delta$. If $\mathrm{LN}$ is not conserved and there are no light sterile neutrinos the neutrino mass sector is completely different from the quark sector but still can be parametrized by the three masses, three mixing angles, the phase $\delta$ and two additional phases, $\alpha_{1,2}$, which are characteristic of Majorana neutrinos. Neutrino oscillation experiments only depend on mass differences, the mixing angles and the LN conserving phase $\delta$, but not on the Dirac/Majorana character of the neutrinos. Thus, present data on neutrino oscillations allow us to determine rather precisely the mass differences, $\Delta m_{21}^{2},\left|\Delta m_{31}^{2}\right|$, and the mixing angles, ${ }^{1} \theta_{12}, \theta_{23}, \theta_{13}$, but little is known on the phase, $\delta$, the sign of $\Delta m_{31}^{2}$ or the absolute neutrino mass scale (characterized, for instance, by the lightest neutrino mass).

However, from the conceptual point of view, the main question is whether neutrinos are Dirac or Majorana, for the symmetries they preserve are different and require quite different descriptions in quantum field theory. In fact, if there are no sterile neutrinos, active neutrinos should necessarily be Majorana neutrinos, LN should not be conserved and the SM should be extended with new particles in order to allow for lepton number violation (LNV). Can one test if neutrino masses are

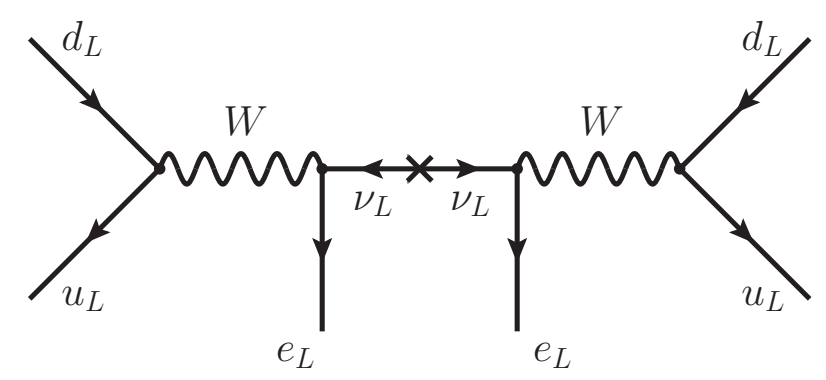

Figure 1: Majorana neutrino mass contribution to $0 v \beta \beta$.

of the Majorana type? It seems very difficult since we have only observed neutrino masses in neutrino flavour oscillations, which conserve total lepton number. LNV neutrino transitions (for instance, a $\mu^{+}$produced by a $v_{\mu}$ from $\mu^{-}$decay) are in principle possible for Majorana neutrinos, but, unless other sources of LNV are present, they are suppressed by $m_{v} / E$ factors and do not enjoy an oscillatory behaviour [9-13]. Alternatively, one can look for LNV decays. Since Majorana neutrinos violate LN, searching for LNV we are indirectly testing the Majorana character of the

\footnotetext{
${ }^{1}$ In the standard parametrization the sign of $\Delta m_{21}^{2}=m_{2}^{2}-m_{1}^{2}$ can be always chosen positive by convention. This is not true for $\Delta m_{31}^{2}=m_{3}^{2}-m_{1}^{2}$ once the mixing angles are taken to vary only in the first quadrant. The solution with $\Delta m_{31}^{2}>0$ is usually named normal hierarchy (NH) while $\Delta m_{31}^{2}<0$ is called inverted hierarchy (IH).
} 


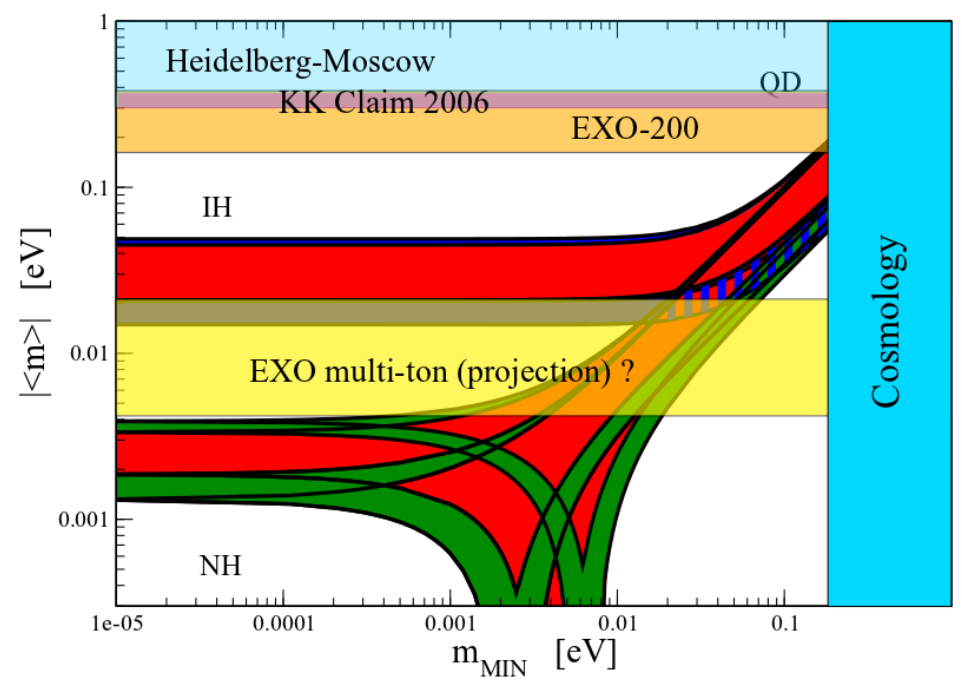

Figure 2: Prospects for testing $0 v \beta \beta$ if induced only by Majorana neutrino masses.

neutrinos. The best candidate is neutrinoless double beta decay $(0 v \beta \beta)$ which is also suppressed by $m_{v}$ but is enhanced with respect to the $\mathrm{LN}$ conserving process (double beta decay with emission of two neutrinos), which has been already observed, by huge phase space factors. In Figure 1 we display the standard Majorana neutrino contribution to $0 v \beta \beta$. Assuming no new physics (NP) beyond the SM and only three Majorana neutrinos the present/future situation can be summarized by plotting the allowed regions in the plane $\left\langle m_{v}\right\rangle-m_{\mathrm{MIN}}$, where $\left\langle m_{v}\right\rangle$ is a combination of masses and mixings relevant in $0 v \beta \beta$ and $m_{\mathrm{MIN}}$ is the lightest neutrino mass. Under these assumptions, present experiments are not able yet to probe neutrino masses. Planned experiments, however, will be able to do it if neutrinos present an IH spectrum and/or $m_{\mathrm{MIN}}$ is above a few tens of meV. If $m_{\mathrm{MIN}}$ is below $10 \mathrm{meV}$ and neutrino masses present a $\mathrm{NH}$ spectrum, it will be very difficult to test $\mathrm{LNV}$ in $0 v \beta \beta$.

On the other hand, the relation between Majorana neutrino masses and $0 v \beta \beta$ is not so direct in general, and there could be new contributions to $0 v \beta \beta$ which could render it observable in planned experiments and perhaps even in upgrades of ongoing ones. In fact, from the symmetry point of view, Majorana masses break any charge carried by $v_{L}$ by two units, while $0 v \beta \beta$ can proceed in different ways depending on the chirality of the emitted electrons, $e_{L} e_{L}, e_{L} e_{R}$ or $e_{R} e_{R}$. Hence, this decay will break any charge carried by $e_{L}$ and $e_{R}$ by $(2,0),(1,1)$ and $(0,2)$ units, respectively. Therefore, Majorana neutrino masses and $0 v \beta \beta$ could have, in principle, a quite different origin. However, as in the SM $v_{L}$ and $e_{L}$ are in the same multiplet, they should carry the same type of lepton number while $e_{L}$ and $e_{R}$ are only linked by the electron mass. This just tells that the connection between the different lepton numbers is valid only up to SM gauge symmetry and/or chirality breaking effects. Moreover, there is a general argument [14] which suggests that if $0 v \beta \beta$ exists there should necessarily be contributions to Majorana neutrino masses induced by the 

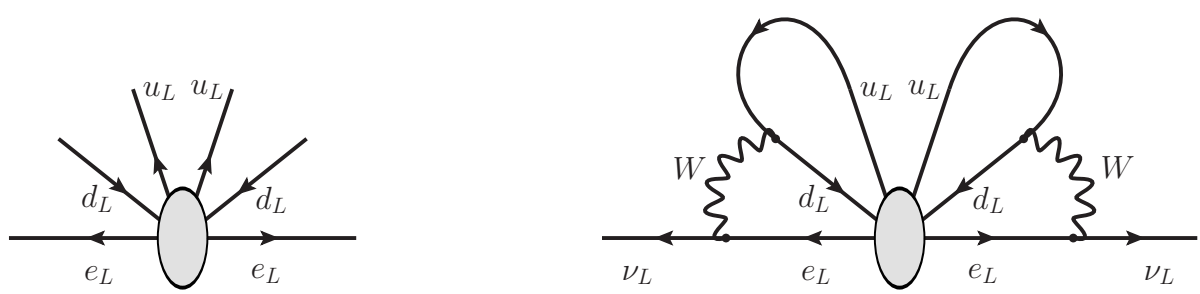

Figure 3: How $0 v \beta \beta$ induces Majorana neutrino masses (at four loops).

$0 v \beta \beta$ interactions (see Figure 3). Nevertheless, these contributions are generated at four loops and are expected to be very small (for a calculation in the low-energy effective theory see [15]).

In view of this discussion it is important to explore the possibility of additional contributions to $0 v \beta \beta$ and their connection with neutrino masses, a scenario to be tested by existing and forthcoming experiments. Since both, neutrino masses and $0 v \beta \beta$, are low energy processes, an effective Lagrangian approach [16-21] is the proper starting point of any model-independent analysis. Several papers [22-25] have followed this approach, considering only effective interactions not involving SM gauge bosons. Here we review a different class of theories where NP does not couple directly to the quark sector ${ }^{2}$, so that the effective interactions involve only leptons and gauge bosons (couplings to gauge bosons are generated whenever the NP is not a SM gauge singlet). In this case it is possible to give a simple classification of the effects that concern us in terms of only three operators, each of which can be generated at tree level by different types of NP [28]. In the unitary gauge these operators give the vertices $v_{L} v_{L}, W e_{R} v_{L}$ and $W W e_{R} e_{R}$ and have dimension 5,7 and 9, respectively. This allows for three scenarios wherein one of the operators is generated at tree level and the others via loops. A simple example exhaustively considered in the literature has tree-level neutrino masses generated via a high-scale see-saw mechanism [29-32], with effective $W e_{R} v_{L}$ and $W W e_{R} e_{R}$ vertices generated radiatively. Here we concentrate on the complementary case where the LNV operators contributing to $0 v \beta \beta$ are generated at tree level whereas neutrino masses are induced radiatively. It is also very important to be able to build renormalizable models which could give rise to the different operators in the effective Lagrangian at low energies. As we will stress, these models are quite interesting because some of the new particles may be accessible to LHC $[33,34]$.

Thus, using the effective Lagrangian approach, in Section 2 we review the classification of the NP contributions to $0 v \beta \beta$ and their connection with neutrino masses [28]. In Section 3 we will discuss some examples of renormalizable completions giving rise to the relevant operators presented in Section 2 [28, 33]. Finally, Section 4 is devoted to our conclusions. Further details of the topics discussed in this talk and a more complete list of references can be found in these papers.

\section{NP Contributions to $0 v \beta \beta$}

The observation of $0 v \beta \beta$ requires LNV, while SM interactions conserve $\mathrm{LN}$. Therefore, new interactions must be added to the SM in order to allow for $0 v \beta \beta$. We illustrate the different

\footnotetext{
${ }^{2}$ Operators involving leptons and quarks with no gauge bosons generate neutrino masses at 1 to 4 loops [25, 26, 15, 27] and may receive enhancements from top Yukawa couplings.
} 


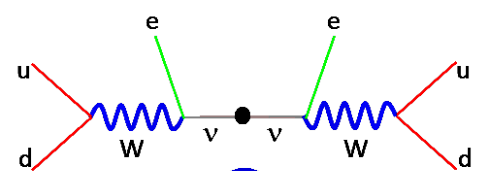

(A)

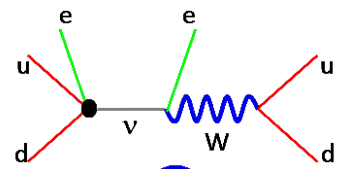

(D)

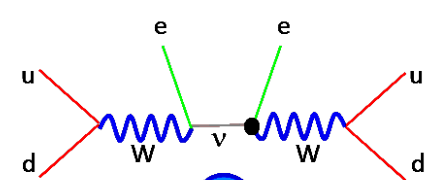

(B)

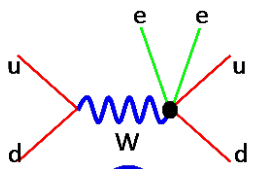

(E)

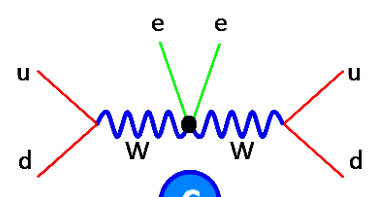

(C)

Figure 4: Classification of possible contributions to $0 v \beta \beta$.

possibilities diagrammatically in Figure 4, where only one LNV vertex is considered at a time. The new interactions are represented by a big dot and the $e$ could be $e_{L}$ or $e_{R}$ depending on the particular vertex. Diagram $A$ is the usual contribution provided by Majorana neutrino masses, diagrams $D-F$ require new interactions involving quarks which will not be considered here anymore. Therefore, we will concentrate on contributions of type $B$ and $C$ and in their relation with neutrino masses (contributions of type $A$ ). Figure 4 represents the low energy form of the different interactions, but they must be obtained preserving the SM gauge symmetries. For this we should use the effective Lagrangian approach which, being very general, still involves some assumptions:

- The SM is a low-energy approximation of a more complete theory.

- The only light particles $(m \lesssim 250 \mathrm{GeV})$ are those of the SM.

Then, the effective Lagrangian can be written as

$$
\mathscr{L}=\mathscr{L}_{\mathrm{SM}}+\sum_{n=5}^{\infty} \sum_{i}\left(\frac{C_{i}^{(n)}}{\Lambda^{n-4}} \mathscr{O}_{i}^{(n)}+\text { h.c. }\right),
$$

where $\mathscr{L}_{\mathrm{SM}}$ is the SM Lagrangian ( $\phi$ and $\ell$ stand for the SM Higgs and left-handed (L) lepton doublets, $e_{R}$ the right-handed (R) charged lepton singlets, whereas flavour indices are suppressed and $Y_{e}$ are the corresponding Yukawa couplings)

$$
\mathscr{L}_{\mathrm{SM}}=i \bar{\ell} \not D \ell+i \overline{e_{R}} \not \supset e_{R}-\left(\bar{\ell} Y_{e} e_{R} \phi+\text { h.c. }\right)+\cdots
$$

and $\mathscr{O}_{i}^{(n)}$ are dimension- $n$ gauge invariant operators built with SM fields, being their effects suppressed by $1 / \Lambda^{n}$, with $\Lambda$ the NP scale.

There are in general many operators $\mathscr{O}_{i}^{(n)}$ (see for instance [22-25]), but if we restrict ourselves to those not involving quarks, the list of relevant, lowest order operators, reduces to only three which, amazingly, can be classified according to the chirality of the emitted electrons, LL, LR, RR, and correspond to the diagrams of type $A-C$ in Figure 4, respectively (for details see [28]). They read 


$$
\begin{array}{ll}
\text { LL : } & \mathscr{O}^{(5)}=\left(\overline{\tilde{\ell}_{\alpha}} \phi\right)\left(\tilde{\phi}^{\dagger} \ell_{\beta}\right)=-v^{2} \overline{v_{\alpha \mathrm{L}}^{\mathrm{c}}} v_{\beta \mathrm{L}}+\ldots, \\
\mathrm{LR}: & \mathscr{O}^{(7)}=\left(\phi^{\dagger} D_{\mu} \tilde{\phi}\right)\left(\phi^{\dagger} \overline{e_{\alpha \mathrm{R}}} \gamma^{\mu} \tilde{\ell}_{\beta}\right)=i \frac{g v^{3}}{\sqrt{2}} W_{\mu}^{-} \overline{e_{\alpha \mathrm{R}}} \gamma^{\mu} v_{\beta \mathrm{L}}^{\mathrm{c}}+\ldots, \\
\mathrm{RR}: & \mathscr{O}^{(9)}=\overline{e_{\alpha \mathrm{R}}} e_{\beta \mathrm{R}}^{\mathrm{c}}\left(\phi^{\dagger} D \tilde{\phi}\right)^{2}=-\frac{g^{2} v^{4}}{2} W_{\mu}^{-} W^{-\mu} \overline{\bar{e}_{\alpha \mathrm{R}}} e_{\beta \mathrm{R}}^{\mathrm{c}}+\ldots,
\end{array}
$$

where $v=\langle\phi\rangle \sim 174 \mathrm{GeV}$ is the SM vacuum expectation value (VEV) and $\tilde{\phi}=i \tau_{2} \phi^{*}, \tilde{\ell}=i \tau_{2} \ell^{c}$ are the conjugate SM doublets with $\tau_{2}$ the weak isospin Pauli matrix. From the interactions (2.3-2.5) one can immediately estimate the amplitudes contributing to $0 v \beta \beta$

$$
\begin{aligned}
\mathrm{LL}: & \mathscr{A}_{0 v \beta \beta}^{(5)} \sim \frac{C_{e e}^{(5)}}{\Lambda p_{\mathrm{eff}}^{2} \nu^{2}}, \\
\mathrm{LR}: & \mathscr{A}_{0 v \beta \beta}^{(7)} \sim \frac{C_{e e}^{(7)}}{\Lambda^{3} p_{\mathrm{eff}} v}, \\
\mathrm{RR}: & \mathscr{A}_{0 v \beta \beta}^{(9)} \sim \frac{C_{e e}^{(9)}}{\Lambda^{5}},
\end{aligned}
$$

with $p_{\text {eff }} \sim 100 \mathrm{MeV}$ the effective momentum exchanged, which is estimated from complete nuclear matrix elements calculations. $0 v \beta \beta$ experiments $^{3}$ (HM,IGEX) give $T_{1 / 2}>1.9 \times 10^{25}$ years, implying

$$
\frac{p_{\text {eff }}}{G_{F}^{2}}\left|\mathscr{A}_{0 v \beta \beta}\right| \lesssim 5 \times 10^{-9}
$$

Imposing this bound on the different terms, we obtain

$$
\begin{array}{ll}
\text { LL : } & \Lambda>10^{11}\left|C_{e e}^{(5)}\right| \mathrm{TeV}, \\
\text { LR : } & \Lambda>106\left|C_{e e}^{(7)}\right|^{1 / 3} \mathrm{TeV}, \\
\mathrm{RR}: & \Lambda>2.7\left|C_{e e}^{(9)}\right|^{1 / 5} \mathrm{TeV} .
\end{array}
$$

By using detailed nuclear matrix elements these estimates do not substantially change.

\subsection{Contribution to $v$ masses}

Once LN is violated by the operators $\mathscr{O}^{(n)}$, neutrino masses (that is $\mathscr{O}^{(5)}$ ) will be sooner or later generated. Thus, $\mathscr{O}^{(7)}$ will give one-loop contributions to neutrino masses while the first contributions to neutrino masses from $\mathscr{O}^{(9)}$ will arise at two loops. Although these contributions cannot be calculated precisely in the effective theory, they can be estimated by naive dimensional analysis. In Table 1 we present the relevant diagrams and estimates for the masses. It is important to notice the presence of charged fermion mass factors, $m_{a}$. As emphasized in the introduction, in the $m_{a} \rightarrow 0$ limit, lepton numbers carried by $v_{L}$ and $e_{L}$ cannot be linked to that carried by $e_{R}$.

\footnotetext{
${ }^{3} \mathrm{EXO}$ has improved it recently in about a factor of 2 .
} 
LL :

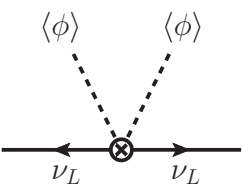

LR :
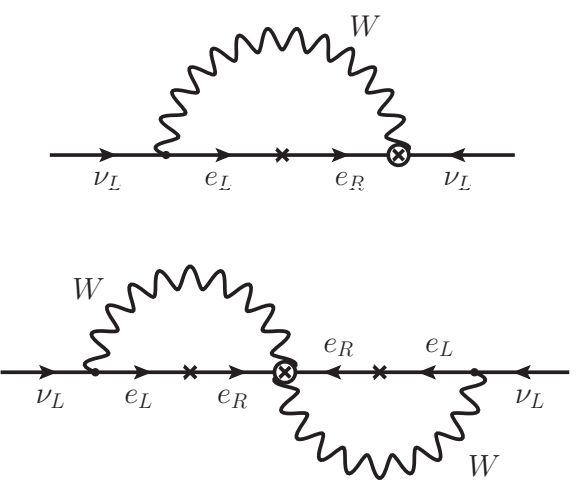

$$
\left(m_{v}\right)_{a b} \sim \frac{v^{2}}{\Lambda} C_{a b}^{(5)}
$$$$
\left(m_{v}\right)_{a b} \sim \frac{v}{16 \pi^{2} \Lambda}\left(m_{a} C_{a b}^{(7)}+m_{b} C_{b a}^{(7)}\right)
$$

$$
\left(m_{v}\right)_{a b} \sim \frac{1}{\left(16 \pi^{2}\right)^{2} \Lambda} m_{a} C_{a b}^{(9)} m_{b}
$$

Table 1: Contribution to neutrino masses from the relevant operators giving rise to $0 v \beta \beta$.

Hence, one mass insertion is needed to generate neutrino masses from $\mathscr{O}^{(7)}$ (which involves $e_{L}$ and $e_{R}$ ) and two mass insertions to generate them from $\mathscr{O}^{(9)}$ (which involves two $e_{R}$ ). The hierarchy of charged lepton masses then translates into a quite characteristic structure of neutrino masses with interesting phenomenological consequences. We will discuss them in the framework of specific models where all the coefficients can be precisely calculated.

Assuming that the dominant contribution to neutrino masses comes from the operators $\mathscr{O}^{(5-9)}$ and the diagrams in Table 1, we can correlate them and the $0 v \beta \beta$ amplitudes

$$
\begin{array}{ll}
\text { LL }: & \mathscr{A}_{0 v \beta \beta}^{(5)} \propto\left(m_{v}\right)_{e e}, \\
\text { LR }: & \mathscr{A}_{0 v \beta \beta}^{(7)} \propto\left(m_{v}\right)_{e e}(4 \pi)^{2} \frac{v^{2}}{\Lambda^{2}} \frac{p_{\mathrm{eff}}}{m_{e}}, \\
\text { RR }: & \mathscr{A}_{0 v \beta \beta}^{(9)} \propto\left(m_{v}\right)_{e e}\left((4 \pi)^{2} \frac{v^{2}}{\Lambda^{2}} \frac{p_{\text {eff }}}{m_{e}}\right)^{2} .
\end{array}
$$

Thus, in general, the standard Majorana neutrino mass contribution to $0 v \beta \beta$ in Figures 1 and $4 \mathrm{~A}$, will be always present together with the new contributions given by the effective operators in Figure 4B-F. The interesting situation occurs when the new contributions dominate over the standard one exchanging a massive Majorana neutrino, otherwise the standard analysis applies. Thus, LR and RR contributions are larger when

$$
\Lambda<4 \pi v \sqrt{\frac{p_{\text {eff }}}{m_{e}}} \sim 30 \mathrm{TeV}
$$

and, therefore, the NP scale is relatively low, and perhaps accessible to LHC.

\section{Renormalizable Completions}

The effective Lagrangian approach is very general and, as seen, just using it one can grasp the 

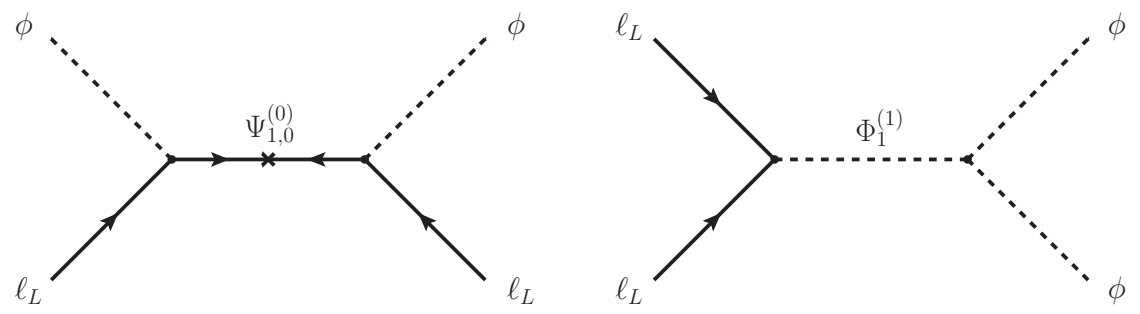

Figure 5: The three see-saw mechanisms generating the operator $\mathscr{O}^{(5)}(\mathrm{LL})$.

main consequences of the different scenarios. However, sometimes it is quite difficult to realize a specific effective Lagrangian with a renormalizable model. Therefore, it is important to check that these effective theories can arise from a consistent renormalizable model and that the estimates obtained in the effective Lagrangian approach are correct. Moreover, in our case, since the scale of NP is not extremely large, perhaps the new particles are accessible at LHC and/or provide interesting effects in lepton flavour violation (LFV) processes, providing additional tests of the proposed mechanisms for $0 v \beta \beta$. Of course, one needs explicit models to address this.

The operators $\mathscr{O}^{(5)}, \mathscr{O}^{(7)}, \mathscr{O}^{(9)}$ can be obtained from renormalizable interactions by adding a variety of new particles. We will denote by $\Phi_{I}^{(Y)}$ scalars with hypercharge $Y$ and weak isospin I. Similarly $\Psi_{I}^{(Y)}$ will be spin $1 / 2$ particles and $X_{I}^{(Y)}$ vector bosons. The different ways of obtaining the operator $\mathscr{O}^{(5)}(\mathrm{LL})$ at tree level have been extensively studied and constitute the three see-saw mechanisms which are represented diagrammatically in Figure 5: (left) exchange of a neutral fermion $\Psi_{0}^{(0)}$ (type I see-saw) or of a fermion triplet $\Psi_{1}^{(0)}$ (type III see-saw); (right) exchange of a scalar triplet $\Phi_{1}^{(1)}$ (type II see-saw). For $\mathscr{O}^{(7)}$ (LR) and $\mathscr{O}^{(9)}$ (RR) there are many more possibilities (they have been classified in [28]). For instance, $\mathscr{O}^{(7)}$ can be obtained adding $\left\{\Psi_{1 / 2}^{(1 / 2)}, \Phi_{1}^{(1)}\right\} ;\left\{\Psi_{0}^{(0)}, \Phi_{0}^{(1)}\right\}, \cdots$, and $\mathscr{O}^{(9)}$ results from integrating out $\left\{\Phi_{0}^{(2)}, \Phi_{1}^{(1)}\right\} ;\left\{\Psi_{1}^{(0)}, \Phi_{1}^{(1)}\right\}, \cdots$. In the case that $0 v \beta \beta$ is induced by $\mathscr{O}^{(7,9)}$ we would like to forbid tree-level Majorana neutrino masses because otherwise they will dominate $0 v \beta \beta$. Models can be supplemented with additional global continuous or discrete symmetries to forbid $\mathscr{O}^{(5)}$. In the following we will present some examples of renormalizable theories giving rise to these operators together with their characteristic phenomenology.

\subsection{An example of LR model}

We start from the set $\left\{\Psi_{1 / 2}^{(1 / 2)}, \Phi_{1}^{(1)}\right\}$. In order to simplify the notation we define $\Phi_{1}^{(1)} \equiv \chi$, a scalar isotriplet of hypercharge 1 , and $\Psi_{1 / 2}^{(1 / 2)} \equiv L^{c}=L_{L}^{c}+L_{R}^{c}$, a lepton isodoublet of hypercharge $1 / 2$ (in terms of its left-handed and right-handed components). A simple way to insure the decoupling of the heavy physics is to assume, as we do, that the heavy fermions are vector-like. This particle content is sufficient to generate $\mathscr{O}^{(7)}$ at tree level, and it is not hard to convince oneself that the relevant graphs must involve the couplings $e_{R} \phi \tilde{L}, \ell L \chi$ and $\phi^{\dagger} \phi^{\dagger} \chi$. However, such a model also allows the coupling $\ell \ell \chi$ and will then generate $\mathscr{O}^{(5)}$ at tree level through the standard type-II see-saw diagram. In order to avoid this we impose a discrete $Z_{2}$ symmetry under which $\chi$ and $L$ are odd and $\ell$ is even; unfortunately, this symmetry also forbids the $\phi^{\dagger} \phi^{\dagger} \chi$ vertex. In order to overcome this difficulty we assume the presence of two light scalar doublets $\phi, \phi^{\prime}$, which are even 


\begin{tabular}{l|cccc} 
& $L_{L a}$ & $L_{R a}$ & $\chi$ & $\phi^{\prime}$ \\
\hline$S U(2)_{L}$ & $\frac{1}{2}$ & $\frac{1}{2}$ & 1 & $\frac{1}{2}$ \\
$U(1)_{Y}$ & $-\frac{1}{2}$ & $-\frac{1}{2}$ & 1 & $\frac{1}{2}$ \\
$Z_{2}$ & - & - & - & -
\end{tabular}

Table 2: Quantum number assignment for the extra fields in a model realizing the $\mathscr{O}^{(7)}$ operator.

and odd under $Z_{2}$, respectively. Moreover, in order to accommodate a generic neutrino mass matrix and also allow for flavour symmetries treating the three families on the same footing, we will assume the presence of 3 heavy vector-like fermion doublets $L_{a}, a=1,2,3$. The complete list of new fields is given in Table 2. The Lagrangian will include all renormalizable couplings preserving these symmetries, noting that the SM fields transform trivially under $Z_{2}$.

Thus, the heavy lepton Lagrangian reads

$$
\mathscr{L}_{\mathrm{H}}^{L}=\overline{L_{a}}\left(i \not D-M_{a}\right) L_{a}+\left\{y_{a b}^{e} \overline{L_{a L}} \phi^{\prime} e_{b R}+y_{a b}^{v} \overline{\tilde{L}_{a L}} \chi \ell_{b}+\text { h.c. }\right\},
$$

where we assumed, without loss of generality, that the heavy lepton mass matrix is diagonal. Once $\phi^{\prime}$ and $\chi$ acquire VEVs the light $e_{R}, \ell$ leptons mix with the $L_{a}$. The low-energy effects of such mixings will be proportional to $y_{a b}^{e}\left\langle\phi^{\prime}\right\rangle / M_{a}$ or $y_{a b}^{v}\langle\chi\rangle / M_{a}$ and can be made as small as experimentally required by increasing the heavy masses $M_{a}$, reducing the couplings $y_{a b}^{e, v}$, or the VEVs $\left\langle\phi^{\prime}\right\rangle,\langle\chi\rangle$ (for a recent review on vector-like leptons see, for instance, [35]). LFV effects can be further suppressed by assuming that the light charged leptons, which get their masses through the SM Higgs mechanism, are aligned along the heavy flavours. This corresponds to taking $y_{a b}^{e}$ diagonal, which may be natural in a larger model.

The scalar potential can be easily arranged to insure a minimum where $\langle\phi\rangle \gg\left\langle\phi^{\prime}\right\rangle,\langle\chi\rangle \neq$ 0 , with $\langle\chi\rangle \simeq-\mu^{*}\left\langle\phi^{\prime}\right\rangle\langle\phi\rangle / m_{\chi}^{2}, \mu$ the trilinear $\phi^{\dagger} \chi \tilde{\phi}^{\prime}$ coupling and $m_{\chi}$ the isotriplet mass (in order to satisfy the limit from electroweak precision data [36, 37] we require $\langle\chi\rangle \lesssim 2 \mathrm{GeV}$ ). We assume negative mass terms for $\phi$ and $\phi^{\prime}$ to trigger the corresponding VEVs, whereas $\chi$ gets a VEV through its mixing with the scalar isodoublets. Otherwise, dimensional couplings in the potential are typically of electroweak order, except for new scalar masses that may be larger. Dimensionless ones stay perturbative, in general ranging within an $\alpha_{E M} \sim 10^{-2}$ factor. Note that in this model LN is explicitly broken by (renormalizable) terms in the scalar potential, in particular by the $\left(\phi^{\dagger} \phi^{\prime}\right)^{2}$ term.

It is important to remark that in this model there cannot be tree-level neutrino masses because: (i) $L_{a}$ are doublets and therefore, they cannot mediate the see-saw of types I-III. (ii) There is no coupling $\chi \ell \ell$ due to the discrete symmetry and hence, no type II see-saw contributions.

\subsubsection{The LR operator for $0 v \beta \beta$}

Given the couplings of the model one can evaluate $C_{a b}^{(7)}$ by using the diagram in Figure 6,

$$
\frac{C_{a b}^{(7)}}{\Lambda^{3}}=-i \frac{\mu y_{c a}^{e *} y_{c b}^{\nu *}}{m_{\chi}^{2} M_{c}^{2}},
$$

where all masses in the $L_{c}$ and $\chi$ multiplets are taken equal. 


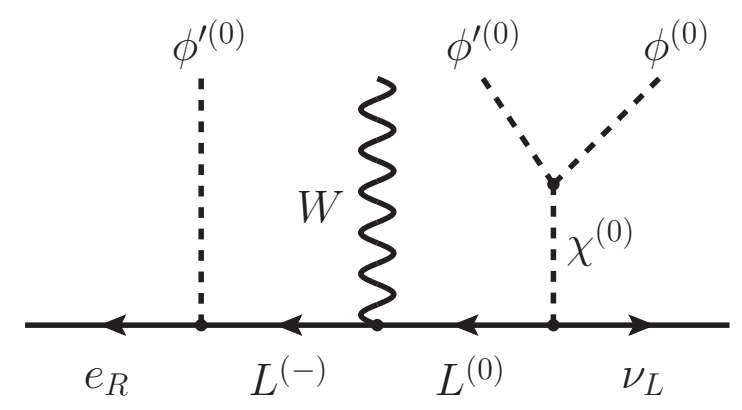

Figure 6: Tree-level diagram contributing to $\mathscr{O}^{(7)}$ in the model proposed.

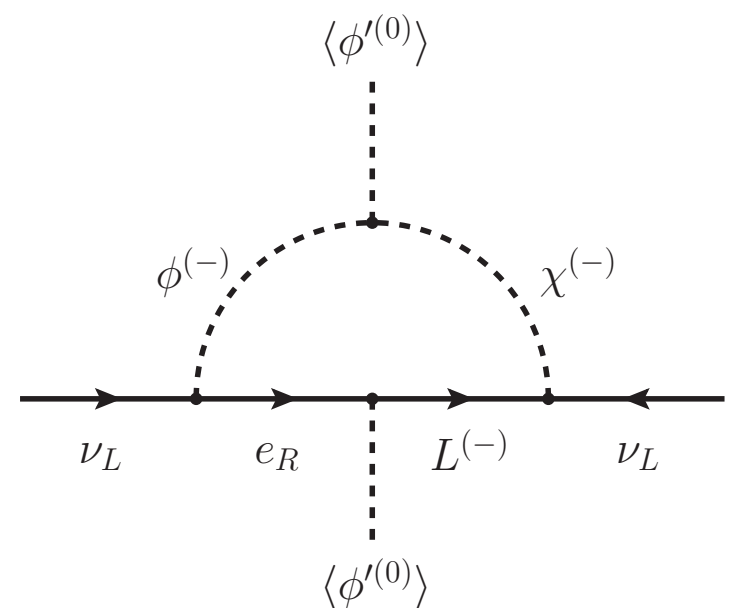

Figure 7: Leading one-loop contribution in the Feynman gauge to neutrino masses in the model generating $\mathscr{O}^{(7)}$.

\subsubsection{The neutrino mass}

Although neutrinos are massless at tree level, as expected from the discussion in Section 2.1, they will acquire a mass at one loop. The dominant contribution can be obtained from the diagram in Figure 7. This is quite different from that used to estimate the mass in the effective theory. This is due to the intricacies of gauge symmetry, but the dominant piece in the limit of small gauge couplings, $g \rightarrow 0$, can be obtained by using only Yukawa couplings as in Figure 7. The result being

$$
\left(m_{v}\right)_{a b} \simeq \frac{v^{\prime 2} \mu}{32 \pi^{2} v}\left(m_{a} y_{c a}^{e *} y_{c b}^{\nu^{*}}+m_{b} y_{c b}^{e *} y_{c a}^{\nu *}\right) \frac{1}{M_{c}^{2}-m_{\chi}^{2}} \log \frac{M_{c}^{2}}{m_{\chi}^{2}}
$$

where $v^{\prime}=\left\langle\phi^{\prime}\right\rangle$ and we have assumed that all other masses are much smaller than $M_{c}$ and $m_{\chi}$. Thus, with only one heavy lepton doublet the neutrino mass matrix has at most rank 2 . With two heavy lepton doublets all three light neutrinos can be massive.

Comparing eqs. (3.3) and eq. (3.2) with the neutrino mass formula in Table 1 for the LR case we see that the general formula roughly applies (take for instance $v^{\prime}=v$ and $\Lambda=M_{c}=m_{\chi}$ ), but by taking different values for the VEVs and the masses of the new particles one can change substantially the numerical values. Since the neutrino masses are generated at one loop and are 
suppressed by only one charged lepton mass they will tend to be too large for masses $m_{\chi}, M_{c}$ just above the electroweak scale and Yukawa couplings of order one. However, by playing with the ratios $v^{\prime} / v$ and $m_{\chi} / M_{c}$ one can obtain additional suppression factors for neutrino masses relative to $0 v \beta \beta$.

\subsubsection{Relevant Phenomenology}

The phenomenology of the model is very rich and can be summarized as follows:

By adjusting $v^{\prime} / v, m_{\chi} / M_{c}$ and the couplings one can enhance the tree-level contributions to $0 v \beta \beta$ enough to be at the reach of the present round of experiments. On the other hand, the neutrino mass matrix has a very characteristic structure, eq. (3.3), but still can accommodate the observed neutrino spectrum. The corresponding neutrino mass constraints together the requirement of a large $0 v \beta \beta$ favour small couplings and relatively light new particles.

Thus, $L_{a}, \chi$ and $\phi^{\prime}$ can be discovered at LHC if light enough ( $\lesssim 800 \mathrm{GeV}$ ), but it depends on the details of the couplings and decay channels. The scalar triplet contains a doubly-charged scalar which will be easy to see if it decays mainly to $e, \mu$, but it will be more complicated to discover if it decays to $\tau, W$ or singly-charged scalars. The production of vector-like leptons $L_{a}$ at LHC has been previously studied [38], with the general conclusion that they can be detected provided their masses are below $\sim 850 \mathrm{GeV}$ for a center of mass $(\mathrm{CM})$ energy of $14 \mathrm{TeV}$ and an integrated luminosity of $100 \mathrm{fb}^{-1}$ [39]. The LHC reach reduces to $\sim 350 \mathrm{GeV}$ for heavy leptons mainly decaying into taus [40].

LFV can be always made small at tree level even though once $\phi^{\prime}$ and $\chi$ acquire VEVs light $e_{R}, \ell$ leptons mix with the heavy ones $L_{a}$. Such mixings and the corresponding phenomenology of heavy vector-like lepton doublets were analyzed long ago in different contexts (for a review see [41, 42]; for updated limits see [35]). The low-energy effects of these mixings are proportional to $y_{a b}^{e}\left\langle\phi^{\prime}\right\rangle / M_{a}$ or $y_{a b}^{v}\langle\chi\rangle / M_{a}$, and can be made as small as experimentally required by increasing the heavy masses $M_{a}$, reducing the couplings $y_{a b}^{e, v}$, or the VEVs $\left\langle\phi^{\prime}\right\rangle,\langle\chi\rangle$. LFV effects can be further suppressed by assuming that the light charged leptons, which get their masses through the SM Higgs mechanism, are aligned along the heavy flavors. This corresponds to taking $y_{a b}^{e}$ diagonal, which may be natural in a larger model.

At one loop LFV through the exchange of heavy leptons and bosons provides the most stringent constraints on this model, but they can be avoided. The most restrictive processes are those involving the muon to electron transition. Then, one can, to a large extent, apply the conclusions from related analyses for the Littlest Higgs model with T-parity [43-46]; the general conclusion is that the heavy flavors must be aligned with the light charged leptons with a precision better than $1-10 \%$ for heavy masses of $\mathscr{O}(\mathrm{TeV})$.

More details on the phenomenology of this model can be found in [28].

\subsection{An example of RR model}

In order to generate $\mathscr{O}^{(9)}$ we can add the scalars $\left\{\Phi_{0}^{(2)}, \Phi_{1}^{(1)}\right\}$ to the SM. For easy notation we will name the doubly-charged scalar $\Phi_{0}^{(2)}=\kappa$ and the triplet scalar $\Phi_{1}^{(1)}=\chi$. Moreover, we will impose a discrete $Z_{2}$ symmetry in order to forbid the $\chi \ell \ell$ coupling, which would provide tree-level neutrino masses once the triplet develops a VEV. This symmetry can be implemented adding a real 


\begin{tabular}{l|ccc} 
& $\chi$ & $\kappa$ & $\sigma$ \\
\hline$S U(2)_{L}$ & 1 & 0 & 0 \\
$U(1)_{Y}$ & 1 & 2 & 0 \\
$Z_{2}$ & - & + & -
\end{tabular}

Table 3: New fields and their quantum numbers in a model realizing the $\mathscr{O}^{(9)}$ operator.

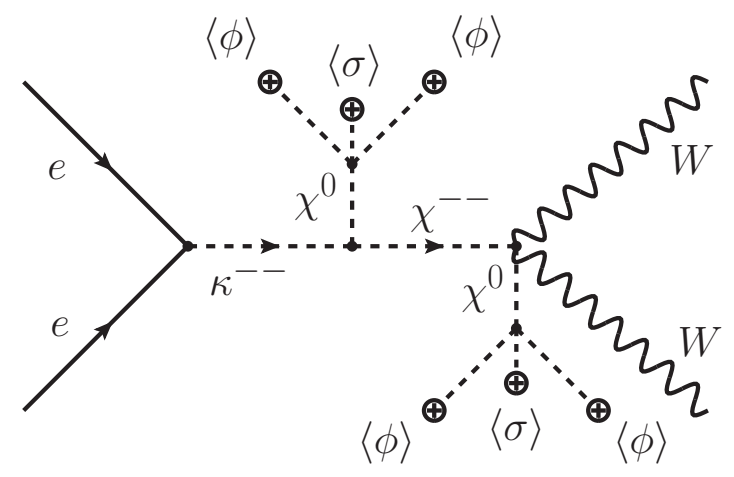

Figure 8: Tree-level diagram contributing to $\mathscr{O}^{(9)}$ in the model proposed.

scalar $\sigma$, odd under $Z_{2}$. This scalar is not really necessary if one allows for a soft breaking of this discrete symmetry, what provides a simpler variation of the model. Other variations are discussed in [33] but most of their phenomenology is shared by the model discussed here. The spectrum of new particles and their quantum numbers are displayed in Table 3, allowing from the new terms in the Lagrangian

$$
\mathscr{L}=g_{\alpha \beta}{\overline{e_{\alpha \mathrm{R}}}}^{\mathrm{c}} e_{\beta \mathrm{R}} \kappa-\mu_{\kappa} \kappa \operatorname{Tr}\left\{\chi^{\dagger} \chi^{\dagger}\right\}-\lambda_{6} \sigma \phi^{\dagger} \chi \tilde{\phi}+\cdots .
$$

This model does not have tree-level neutrino masses because: (i) There are no new fermions in the spectrum and therefore, there cannot be type I-III see-saw neutrino mass contributions. (ii) There is no coupling $\chi \ell \ell$ due to the discrete symmetry and hence, type II see-saw neutrino masses do not arise either.

\subsubsection{The RR operator for $0 v \beta \beta$}

From the Lagrangian in eq. (3.4) and the diagram in Figure 8, one can obtain the $\mathscr{O}^{(9)}$ coefficient in the corresponding effective Lagrangian

$$
\frac{C_{a b}^{(9)}}{\Lambda^{5}}=-i \frac{4 v_{\chi}^{2} \mu_{\kappa}}{m_{\kappa}^{2} m_{\chi}^{2} v^{4}} g_{a b}^{*},
$$

where the result is expressed in terms of the SM VEV, $v$, and the triplet one, $v_{\chi} \approx-\lambda_{6}\langle\sigma\rangle\langle\phi\rangle^{2} / m_{\chi}^{2}$, as well as the new scalar masses. 


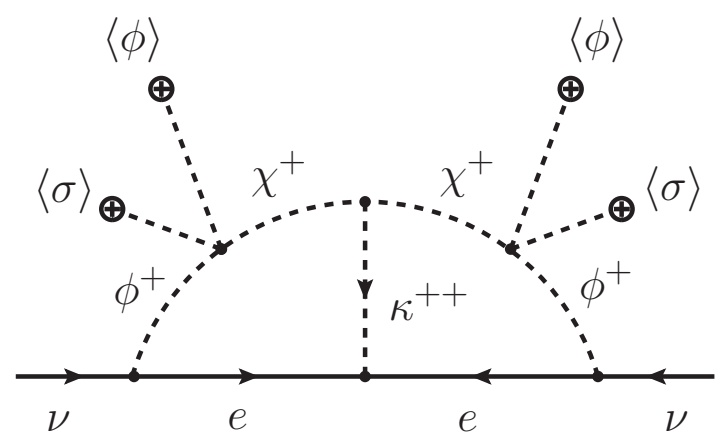

Figure 9: Leading two-loop contribution in the Feynman gauge to neutrino masses in the model generating $\mathscr{O}^{(9)}$.

\subsubsection{The neutrino mass}

As expected, in this case the neutrino mass arises at two loops. The dominant diagram is depicted in Figure 9. The complete calculation, including gauge contributions, can be found in [33]:

$$
\left(m_{v}\right)_{\alpha \beta}=\frac{\mu_{\kappa} v_{\chi}^{2}}{2(2 \pi)^{4} v^{4}} m_{\alpha} g_{\alpha \beta}^{*} m_{\beta} I_{v}
$$

where $I_{v}$ is an order 1 dimensionless function of the new scalar masses and the $W$ mass. The form of $\left(m_{v}\right)_{\alpha \beta}$ agrees with the estimate in Table 1 but contains more parameters which can be used to adjust the neutrino mass matrix elements to fit the observed neutrino spectrum, while keeping $0 v \beta \beta$ at a measurable level.

\subsection{3 $\mathrm{LFV}$ in the RR model}

The exchange of the doubly-charged scalar singlet gives tree-level three-body decays for the SM charged leptons, which do not conserve family lepton number $\left(\ell_{a}^{-} \rightarrow \ell_{b}^{+} \ell_{c}^{-} \ell_{d}^{-}\right.$in Figure 10 and similar diagrams for $\mu^{-}$decay). There are very strong experimental limits on the branching ratios of these processes, and since their amplitudes are proportional to the product $g_{a b} g_{c d}^{*}$, one can set stringent constraints on these couplings. From $\operatorname{BR}\left(\mu^{-} \rightarrow e^{+} e^{-} e^{-}\right)<1.0 \times 10^{-12}$ one obtains

$$
\left|g_{\mu e} g_{e e}^{*}\right|<2.3 \times 10^{-5}\left(m_{\kappa} / \mathrm{TeV}\right)^{2},
$$

whereas $\operatorname{BR}\left(\tau^{-} \rightarrow e^{+} \mu^{-} \mu^{-}\right)<1.7 \times 10^{-8}$ implies

$$
\left|g_{\tau e} g_{\mu \mu}^{*}\right|<0.007\left(m_{\kappa} / \mathrm{TeV}\right)^{2} .
$$

There are also bounds on other muon and tau LFV decay channels $(\mu \rightarrow e \gamma, \tau \rightarrow e \gamma, \tau \rightarrow e e e$, $\mu-e$ conversion in nuclei, $\cdots)$, but for the time being these two processes provide the strongest constraints on the model if one fits the observed neutrino masses allowing for large additional contributions to $0 v \beta \beta$. 


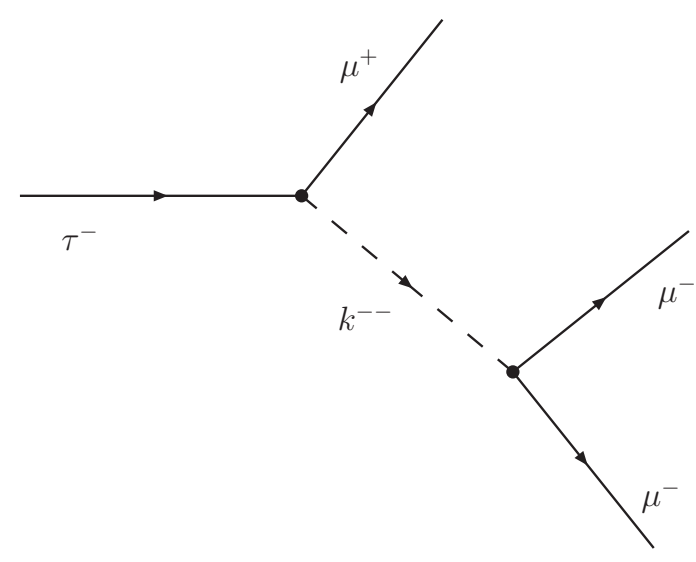

Figure 10: Diagram giving LFV decays.

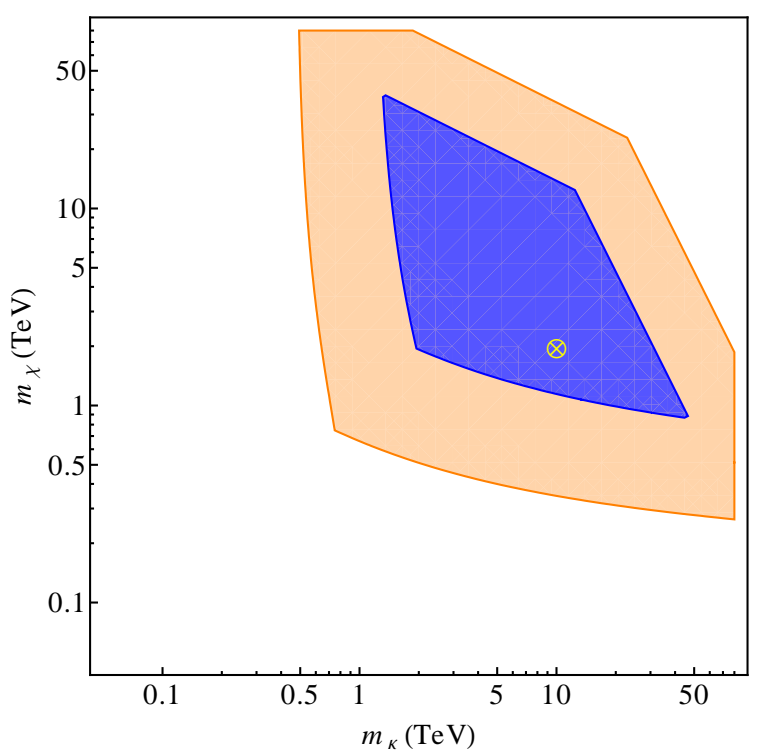

Figure 11: Limits on the masses of the two doubly-charged scalars of the model (see text for details).

\subsubsection{Indirect constraints on doubly-charged scalars}

Since we want to adjust the neutrino masses, have a sizable $0 v \beta \beta$ and satisfy LFV constraints, the model is quite restricted unless one goes to parameter regions where perturbativity does not hold. In particular the doubly-charged scalar masses are quite constrained: if the scalar masses are too large then $0 v \beta \beta$ and neutrino masses will be too small and if the scalar masses are too small the neutrino masses will be too large, LFV limits will be problematic, as well as the bounds from LEP and LHC.

We can visualize the constraints on the scalar masses with the plot in Figure 11, where we show the projection on the $m_{\kappa}-m_{\chi}$ plane of the allowed parameter space region by assuming perturbative unitarity and $\mu_{\kappa}<20 \mathrm{TeV}$. The blue, darker (orange, lighter) areas correspond to $v_{\chi}=2(5) \mathrm{GeV}$. The cross stand for a reference point $m_{\kappa}=10 \mathrm{TeV}, m_{\chi}=2 \mathrm{TeV}$ (and $v_{\chi}=2 \mathrm{GeV}$, $\mu_{\kappa}=15 \mathrm{TeV}$, with $g_{e e}=1$ and $\left.g_{e \mu}=0.001\right)$. 


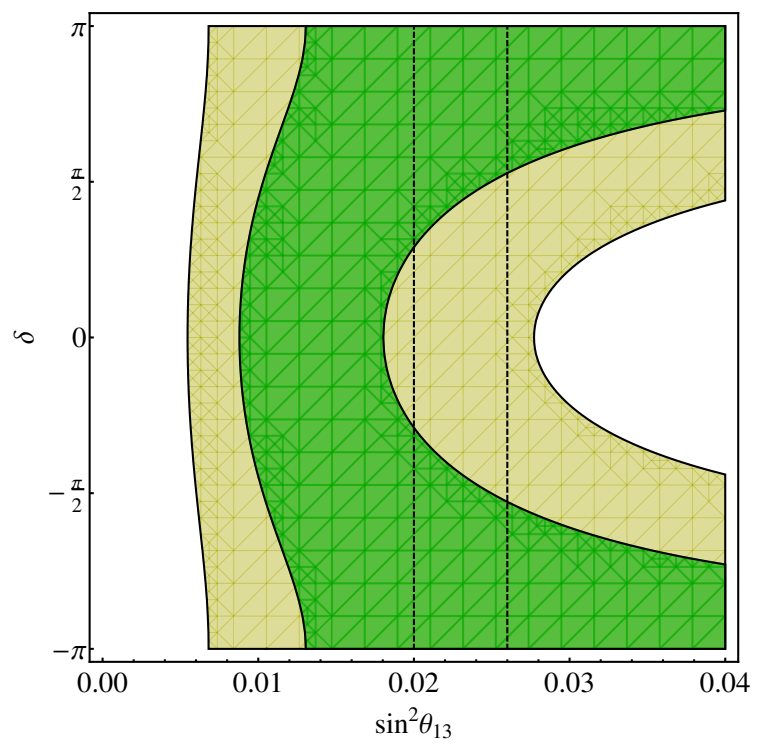

Figure 12: Allowed regions in the plane $\sin ^{2} \theta_{13}-\delta$ when $\left|m_{e e}\right|,\left|m_{e \mu}\right| \ll 0.01 \mathrm{eV}$ (see text).

\subsubsection{Constraints on the $v$ mass matrix}

The particular structure of the neutrino mass matrix $\left(m_{v}\right)_{a b} \propto m_{a} g_{a b}^{*} m_{b}$ together with the limits on $g_{a b}$ coming from LFV processes almost fix the structure of the neutrino mass matrix. Indeed, the requirement of large $0 v \beta \beta$ implies a relatively large $g_{e e}$ and scalar masses relatively low, but on the other hand, $\left(m_{v}\right)_{e e}$ is highly suppressed by the factor $m_{e}^{2}$, while $\left(m_{v}\right)_{e \mu}$ is suppressed because so is $g_{e \mu}$ by the $\mu \rightarrow 3 e$ bound. Thus, taking into account all these limits the neutrino mass matrix must fulfill

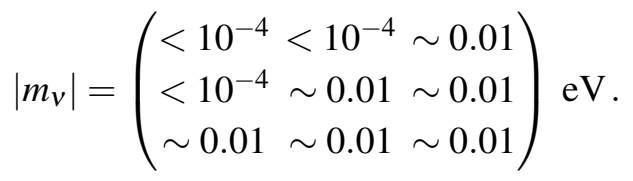

What means that only the NH can be accommodated. Moreover, this structure also provides two predictions:

- One for the lightest neutrino mass, $m_{\mathrm{MIN}}=m_{1} \sim 0.004 \mathrm{eV}$, and

- Other for $\sin ^{2} \theta_{13}$ and $\delta, \sin ^{2} \theta_{13} \gtrsim 0.008$.

In Figure 12 we present the allowed $\sin ^{2} \theta_{13}-\delta$ region for $\left|\left(m_{v}\right)_{e e, e \mu}\right|=0$. The green, darker region is obtained when measured mixings and mass differences (except $\sin \theta_{13}$ ) are varied within $1 \sigma$; while the yellow, lighter one is obtained by varying them within $3 \sigma$ (we use values from the global fit performed in Ref. [47]). For comparison, we also draw the recent measurement of $\sin ^{2}\left(\theta_{13}\right)[48-52]\left(\sin ^{2}\left(\theta_{13}\right)=0.023 \pm 0.003\right.$, dashed lines). From the figure, it seems that large values of $|\delta|$ are somehow favoured by the model. 


\section{Conclusions}

We have used the effective field theory language to classify NP contributions to $0 v \beta \beta$ involving operators without quarks. Charged lepton chiralities, the operator dimension and the order at which $m_{v}$ should appear are linked for the lowest order operators:

- For $e_{L} e_{L}: 0 v \beta \beta$ appears at dimension 5 and $m_{v}$ at tree-level.

- For $e_{L} e_{R}: 0 v \beta \beta$ appears at dimension 7, inducing $m_{v}$ at one loop.

- For $e_{R} e_{R}: 0 v \beta \beta$ appears at dimension 9, being $m_{v}$ induced at two loops.

Hence, it is possible to have a sizable $0 v \beta \beta$ while keeping $m_{v}$ small (loop suppressed).The structure of the neutrino mass matrix is in general constrained, and some of the parameters can be predicted. Although often the models are complicated and tightly restricted, they share a rich phenomenology affecting LFV processes and LHC searches, especially if a doubly-charged scalar is discovered.

\section{Acknowledgments}

This work has been supported in part by the Ministry of Economy and Competitiveness, under the grant numbers FPA2006-05294, FPA2010-17915 and FPA2011-23897, by the Junta de Andalucía grants FQM 101 and FQM 6552, by the "Generalitat Valenciana" grant PROMETEO/2009/128, and by the U.S. Department of Energy grant No. DE-FG03-94ER40837. A.A. is supported by the MICINN under the FPU program.

\section{References}

[1] Particle Data Group Collaboration, J. Beringer et al., Review of Particle Physics (RPP), Phys.Rev. D86 (2012) 010001 [InSP IRE].

[2] R. Mohapatra, S. Antusch, K. Babu, G. Barenboim, M.-C. Chen, et al., Theory of neutrinos: A White paper, Rept.Prog.Phys. 70 (2007) 1757-1867, [arXiv: hep-ph/ 0510213 ] [InSP IRE].

[3] M. C. Gonzalez-Garcia and M. Maltoni, Phenomenology with Massive Neutrinos, Phys. Rept. 460 (2008) 1-129, [arXiv: 0704.1800 ].

[4] M. Gonzalez-Garcia, M. Maltoni, J. Salvado, and T. Schwetz, Global fit to three neutrino mixing: critical look at present precision, JHEP 1212 (2012) 123, [arXiv: 1209.3023] [InSP IRE].

[5] G. Fogli, E. Lisi, A. Marrone, D. Montanino, A. Palazzo, et al., Global analysis of neutrino masses, mixings and phases: entering the era of leptonic CP violation searches, Phys.Rev. D86 (2012) 013012, [arXiv: 1205.5254] [InSPIRE].

[6] D. Forero, M. Tortola, and J. Valle, Global status of neutrino oscillation parameters after Neutrino-2012, Phys.Rev. D86 (2012) 073012, [arXiv: 1205 . 4018] [InSPIRE].

[7] P. Machado, H. Minakata, H. Nunokawa, and R. Zukanovich Funchal, Combining Accelerator and Reactor Measurements of $\theta_{13}$ : The First Result, JHEP 1205 (2012) 023, [arXiv:1111.3330] [InSPIRE]. 
[8] J. Bergstrom, Bayesian evidence for non-zero $\theta_{13}$ and CP-violation in neutrino oscillations, JHEP 1208 (2012) 163, [arXiv: 1205.4404 ] [InSPIRE].

[9] B. Pontecorvo, Mesonium and anti-mesonium, Sov.Phys.JETP 6 (1957) 429 [InSP IRE].

[10] L. Li and F. Wilczek, PHYSICAL PROCESSES INVOLVING MAJORANA NEUTRINOS, Phys.Rev. D25 (1982) 143 [InSPIRE].

[11] J. Schechter and J. Valle, Neutrino Oscillation Thought Experiment, Phys.Rev. D23 (1981) 1666 [InSPIRE].

[12] J. Bernabeu and P. Pascual, CP PROPERTIES OF THE LEPTONIC SECTOR FOR MAJORANA NEUTRINOS, Nucl.Phys. B228 (1983) 21 [InSP IRE].

[13] P. Langacker and J. Wang, Neutrino anti-neutrino transitions, Phys.Rev. D58 (1998) 093004, [arXiv:hep-ph/9802383] [InSPIRE].

[14] J. Schechter and J. Valle, Neutrinoless Double beta Decay in SU(2) x U(1) Theories, Phys.Rev. D25 (1982) 2951 [InSP IRE].

[15] M. Duerr, M. Lindner, and A. Merle, On the Quantitative Impact of the Schechter-Valle Theorem, JHEP 1106 (2011) 091, [arXiv: 1105.0901] [InSP IRE].

[16] S. R. Coleman, J. Wess, and B. Zumino, Structure of phenomenological Lagrangians. 1, Phys. Rev. 177 (1969) 2239-2247.

[17] S. Weinberg, Phenomenological Lagrangians, Physica A96 (1979) 327 [InSP IRE]. Festschrift honoring Julian Schwinger on his 60th birthday.

[18] S. Weinberg, Effective Gauge Theories, Phys.Lett. B91 (1980) 51 [InSP IRE].

[19] J. Polchinski, Renormalization and Effective Lagrangians, Nucl.Phys. B231 (1984) 269-295 [InSPIRE].

[20] H. Georgi, Effective field theory, Ann. Rev. Nucl. Part. Sci. 43 (1993) 209-252.

[21] J. Wudka, Electroweak effective Lagrangians, Int.J.Mod.Phys. A9 (1994) 2301-2362, [arXiv:hep-ph/9406205] [InSPIRE].

[22] K. S. Babu and C. N. Leung, Classification of effective neutrino mass operators, Nucl. Phys. B619 (2001) 667-689, [arXiv: hep-ph/0106054].

[23] K. Choi, K. S. Jeong, and W. Y. Song, Operator analysis of neutrinoless double beta decay, Phys. Rev. D66 (2002) 093007, [arXiv: hep-ph/ 0207180 ] [InSPIRE].

[24] J. Engel and P. Vogel, Effective operators for double beta decay, Phys.Rev. C69 (2004) 034304, [arXiv:nucl-th/0311072] [InSPIRE].

[25] A. de Gouvea and J. Jenkins, A Survey of Lepton Number Violation Via Effective Operators, Phys. Rev. D77 (2008) 013008, [arXiv: 0708 .1344] [InSPIRE].

[26] K. Babu and J. Julio, Two-Loop Neutrino Mass Generation through Leptoquarks, Nucl.Phys. B841 (2010) 130-156, [arXiv:1006.1092] [InSPIRE].

[27] K. Babu and J. Julio, Radiative Neutrino Mass Generation through Vector-like Quarks, Phys.Rev. D85 (2012) 073005, [arXiv:1112.5452] [InSPIRE].

[28] F. del Aguila, A. Aparici, S. Bhattacharya, A. Santamaria, and J. Wudka, Effective Lagrangian approach to neutrinoless double beta decay and neutrino masses, JHEP 1206 (2012) 146, [arXiv:1204.5986] [InSPIRE]. 
[29] P. Minkowski, $\mu \rightarrow$ e $\gamma$ at a Rate of One Out of 1-Billion Muon Decays?, Phys.Lett. B67 (1977) 421 [InSPIRE].

[30] M. Gell-Mann, P. Ramond, and R. Slansky, Complex Spinors and Unified Theories, Conf.Proc. C790927 (1979) 315-321 [InSP IRE]. Published in Supergravity, P. van Nieuwenhuizen and D.Z. Freedman (eds.), North Holland Publ. Co., 1979.

[31] T. Yanagida, Horizontal Symmetry and Masses of Neutrinos, Conf.Proc. C7902131 (1979) 95 [InSPIRE].

[32] R. N. Mohapatra and G. Senjanovic, Neutrino Mass and Spontaneous Parity Violation, Phys.Rev.Lett. 44 (1980) 912 [InSPIRE].

[33] F. del Aguila, A. Aparici, S. Bhattacharya, A. Santamaria, and J. Wudka, A realistic model of neutrino masses with a large neutrinoless double beta decay rate, JHEP 1205 (2012) 133, [arXiv:1111.6960] [InSPIRE].

[34] F. del Aguila, M. Chala, A. Santamaria, and J. Wudka, Discriminating between lepton number violating scalars using events with four and three charged leptons at the LHC, [arXiv:1305.3904] [InSPIRE].

[35] F. del Aguila, J. de Blas, and M. Perez-Victoria, Effects of new leptons in Electroweak Precision Data, Phys.Rev. D78 (2008) 013010, [arXiv: 0803.4008 ] [InSP IRE].

[36] Particle Data Group Collaboration, K. Nakamura et al., Review of particle physics, J.Phys.G G37 (2010) 075021 [InSPIRE].

[37] F. del Aguila, J. Aguilar-Saavedra, J. . de Blas, and M. Perez-Victoria, Electroweak constraints on see-saw messengers and their implications for LHC, [arXiv: 0806.1023 ] [InSP IRE]. Published in the Proceedings of "43rd Rencontres de Moriond on Electroweak Interactions and Unified Theories", 1-8 Mar 2008, La Thuile, Italy.

[38] F. del Aguila, L. Ametller, G. L. Kane, and J. Vidal, Vector-Like Fermion and Standard Higgs Production at Hadron Colliders, Nucl.Phys. B334 (1990) 1 [InSP IRE].

[39] J. Aguilar-Saavedra, Heavy lepton pair production at LHC: Model discrimination with multi-lepton signals, Nucl.Phys. B828 (2010) 289-316, [arXiv: 0905.2221 ] [InSP IRE].

[40] F. del Aguila, A. Carmona, and J. Santiago, Tau Custodian searches at the LHC, Phys.Lett. B695 (2011) 449-453, [arXiv:1007.4206] [InSPIRE].

[41] M. Raidal, A. van der Schaaf, I. Bigi, M. Mangano, Y. K. Semertzidis, et al., Flavour physics of leptons and dipole moments, Eur.Phys.J. C57 (2008) 13-182, [arXiv:0801.1826] [InSPIRE].

[42] T. Feldmann, Lepton Flavour Violation Theory, PoS BEAUTY2011 (2011) 017, [arXiv:1105.2139] [InSPIRE].

[43] M. Blanke, A. J. Buras, B. Duling, A. Poschenrieder, and C. Tarantino, Charged Lepton Flavour Violation and $(\mathrm{g}-2)(\mathrm{mu})$ in the Littlest Higgs Model with T-Parity: A Clear Distinction from Supersymmetry, JHEP 0705 (2007) 013, [arXiv : hep-ph / 0702136 ] [InSP IRE].

[44] F. del Aguila, J. Illana, and M. Jenkins, Precise limits from lepton flavour violating processes on the Littlest Higgs model with T-parity, JHEP 0901 (2009) 080, [arXiv: 0811.2891 ] [InSP IRE].

[45] T. Goto, Y. Okada, and Y. Yamamoto, Tau and muon lepton flavor violations in the littlest Higgs model with T-parity, Phys.Rev. D83 (2011) 053011, [arXiv:1012.4385] [InSP IRE]. 
[46] F. del Aguila, J. Illana, and M. Jenkins, Muon to electron conversion in the Littlest Higgs model with T-parity, JHEP 1009 (2010) 040, [arXiv: 1006.5914 ] [InSP IRE].

[47] T. Schwetz, M. Tortola, and J. Valle, Where we are on $\theta_{13}$ : addendum to 'Global neutrino data and recent reactor fluxes: status of three-flavour oscillation parameters', New J.Phys. 13 (2011) 109401, [arXiv:1108.1376] [InSPIRE].

[48] The MINOS Collaboration, P. Adamson et al., Measurement of the neutrino mass splitting and flavor mixing by MINOS, Phys.Rev.Lett. 106 (2011) 181801, [arXiv:1103.0340] [InSP IRE].

[49] T2K Collaboration, K. Abe et al., Indication of Electron Neutrino Appearance from an Accelerator-produced Off-axis Muon Neutrino Beam, Phys.Rev.Lett. 107 (2011) 041801, [arXiv:1106.2822] [InSPIRE].

[50] DOUBLE-CHOOZ Collaboration, Y. Abe et al., Indication for the disappearance of reactor electron antineutrinos in the Double Chooz experiment, Phys.Rev.Lett. 108 (2012) 131801, [arXiv:1112.6353] [InSPIRE].

[51] DAYA-BAY Collaboration, F. An et al., Observation of electron-antineutrino disappearance at Daya Bay, Phys.Rev.Lett. 108 (2012) 171803, [arXiv:1203.1669] [InSPIRE].

[52] RENO Collaboration, J. Ahn et al., Observation of Reactor Electron Antineutrino Disappearance in the RENO Experiment, Phys.Rev.Lett. 108 (2012) 191802, [arXiv: 1204 . 062 6] [InSP IRE]. 